\title{
Increase in blood glucose level and incidence of diabetic ketoacidosis in children with type 1 diabetes mellitus in the Daegu-Gyeongbuk area during the coronavirus disease 2019 (COVID-19) pandemic: a retrospective cross-sectional study
}

\author{
Mi Seon Lee, Rosie Lee, Cheol Woo Ko, Jung Eun Moon \\ Department of Pediatrics, School of Medicine, Kyungpook National University, Kyungpook National University Hospital, Daegu, Korea
}

Background: The coronavirus disease 2019 (COVID-19) outbreak in the Daegu-Gyeongbuk area in 2020 has caused difficulties in the daily life and hospital care of children with type 1 diabetes mellitus (T1DM). We detected an increase in blood sugar levels in these children and the number of patients hospitalized with more severe diabetic ketoacidosis (DKA) compared to those before COVID-19. Methods: This single-center study was conducted at Kyungpook National University Children's Hospital. The following patient groups were included; 45 returning patients diagnosed with T1DM and undergoing insulin treatment for more than 2 years and 20 patients newly diagnosed with T1DM before and after COVID-19 were selected by age matching. Returning patients before and after the outbreak were selected, and changes in hemoglobin $\mathrm{A} 1 \mathrm{c}(\mathrm{HbA1c})$ levels were retrospectively reviewed. The $\mathrm{HbA} 1 \mathrm{c}$ levels and severity of symptoms in newly diagnosed patients during hospitalization were examined.

Results: HbA1c levels in returning patients with T1DM were significantly increased after COVID-19 (before, 7.70\% $\pm 1.38 \%$ vs. after, $8.30 \% \pm 2.05 \% ; p=0.012$ ). There were 10 and 10 newly diagnosed patients before and after COVID-19, respectively. The proportion of patients with drowsiness and dyspnea at the time of admission was higher after COVID-19 than before (before, 2 of $10 \mathrm{vs.} \mathrm{after,} 4$ of 10). The HbA1c levels were higher in newly diagnosed patients hospitalized after COVID-19 than before (before, $11.15 \%$ vs. after, 13.60\%; $p=0.036)$.

Conclusion: Due to COVID-19 in the Daegu-Gyeongbuk area, there was an increase in blood glucose levels in children with T1DM and in the incidence of severe DKA in newly diagnosed diabetes mellitus patients.

Keywords: COVID-19; Diabetic ketoacidosis; Type 1 diabetes mellitus

\section{Introduction}

In December 2019, atypical pneumonia of unknown cause was first reported in Wuhan, Hubei Province, China [1-3]. In January
2020, the World Health Organization termed this condition as coronavirus disease 2019 (COVID-19), which began to spread rapidly, and eventually, it was declared a pandemic on March 11, 2020 [4].

Received: June 9, 2021 • Revised: August 2, 2021 • Accepted: August 5, 2021

Corresponding author: Jung Eun Moon, MD, PhD

Department of Pediatrics, School of Medicine, Kyungpook National University, Kyungpook National University Hospital, 807 Hoguk-ro, Buk-gu, Daegu 41404 , Korea

Tel: +82-53-200-5704 • Fax: +82-53-425-6683•E-mail: subuya@daum.net 
In Korea, the first imported case of COVID-19 was confirmed on January 20, 2020 [5]. After the first case in the Daegu-Gyeongbuk area was reported on February 18, 2020, there was a rapid increase in the number of patients, which was the first large outbreak in countries other than China [6,7]. Consequently, difficulties in daily life and hospital care in the Daegu-Gyeongbuk area became prominent.

It has been reported that COVID-19 has led to a high mortality rate among adult patients with diabetes mellitus (DM) [8-10]. However, no severe cases of COVID-19 requiring hospitalization have been reported among children and adolescent patients with type $1 \mathrm{DM}$ (T1DM) [11]. The International Society for Pediatric and Adolescent Diabetes stated that diabetic care should be provided while avoiding hospitalization and emergency room visits to prevent infection [12]. However, we observed an increase in overall blood glucose levels compared to the levels before COVID-19 in patients with T1DM in the Daegu-Gyeongbuk area and also encountered new patients who were hospitalized with more severe diabetic ketoacidosis (DKA). This study aimed to investigate the changes in blood glucose levels and clinical manifestations in pediatric patients with T1DM in the Daegu-Gyengbuk area where there was a large outbreak of COVID-19.

\section{Methods}

Ethical statements: This study was approved by the Institutional Review Board (IRB) of Kyungpook National University Chilgok Hospital (IRB No: 2020-09-023) in accordance with the Declaration of Helsinki. Informed consent was waived by the IRB due to the use of anonymized data and the retrospective design.

\section{Subjects and methods}

The study subjects were children aged $<19$ years diagnosed with T1DM at Kyungpook National University Children's Hospital in the Daegu-Gyeongpuk area. A total of 65 patients, including 45 returning patients, 10 newly diagnosed patients during the COVID-19 outbreak, and 10 age-matched patients before the COVID-19 outbreak were included in the control group. A retrospective chart review was conducted. For returning patients undergoing insulin treatment for more than 2 years, patients who had visit records between 3 and 6 months before and after the outbreak were selected. The parameters (age, sex, and changes in hemoglobin $\mathrm{Alc}$ [HbA1c] and body mass index $[\mathrm{BMI}])$ before and after COVID-19 were compared. HbAlc levels were analyzed between the similar age and pubertal groups. The age of the patients was di- vided into $0-4,5-9,10-14,15-18$ years old, and was defined as pubertal age from 10 years of age for girls and 11 years of age for boys. For newly diagnosed patients, 10 new patients who were hospitalized after the COVID-19 outbreak were examined for $\mathrm{HbAlc}$ levels and severity of symptoms at hospitalization. As a control group of newly diagnosed patients, a total of 10 agematched patients with T1DM who visited our hospital 4 years prior to the COVID-19 outbreak were selected. Clinical signs reflecting DKA severity at the time of admission, altered mental status, presence of dyspnea, and laboratory parameters such as $\mathrm{HbAlc}$ levels, initial serum glucose, venous blood gas analysis $\left(\mathrm{pH}, \mathrm{pCO}_{2}\right.$, $\mathrm{HCO}_{3}^{-}$), hemoglobin, blood urea nitrogen, and creatinine were assessed. The severity criteria were as follows: the biochemical criteria for the diagnosis of DKA were blood glucose level of $>200$ $\mathrm{mg} / \mathrm{dL}$, venous $\mathrm{pH}$ of $<7.3$, serum bicarbonate level of $<15$ $\mathrm{mmol} / \mathrm{L}$, and ketonemia or ketonuria of $\geq 2+$. The severity of DKA was categorized based on the degree of acidosis as follows: (1) mild: venous $\mathrm{pH}$ of $<7.3$ or serum bicarbonate level of $<15$ $\mathrm{mmol} / \mathrm{L}$; (2) moderate: $\mathrm{pH}$ of $<7.2$, serum bicarbonate level of $<10 \mathrm{mmol} / \mathrm{L}$; and (3) severe: $\mathrm{pH}$ of $<7.1$, serum bicarbonate level of $<5 \mathrm{mmol} / \mathrm{L}$ [13].

\section{Statistical analysis}

Continuous values are presented as the mean \pm standard deviation. Among the returning patients, the differences in BMI and $\mathrm{HbAlc}$ between the two groups were analyzed using the $t$-test. Due to the small number of newly diagnosed T1DM patients, the distributions of DKA severity, age, sex, $\mathrm{HbAlc}$, glucose, $\mathrm{pH}$, and bicarbonate levels were compared between the groups using Wilcoxon rank-sum tests; the results are reported as medians. All statistical analyses were conducted using IBM SPSS version 20.0 (IBM Corp., Armonk, NY, USA). Statistical significance was set at $p<0.05$.

\section{Results}

\section{Changes in body mass index and hemoglobin A1c levels in returning patients with type 1 diabetes mellitus}

There were 45 subjects with T1DM, consisting of 22 females and 23 males (1:1 ratio), with a mean age of $15.8 \pm 6.13$ years. There were one patient aged 0 to 4 years, five patients aged 5 to 9 years, 21 patients aged 10 to 14 years, and 18 patients aged 15 to 18 years. As baseline characteristics, initial random glucose was $413.53 \pm 202.62 \mathrm{mg} / \mathrm{dL}$ and C-peptide was $0.65 \pm 0.56 \mathrm{ng} / \mathrm{mL}$ (range, $0.4-2.2 \mathrm{ng} / \mathrm{mL}$ ). At least one autoantibody was identified in 37 of 45 patients (82.2\%). Anti-islet cell antibody (ICA) was identified in two patients (4.4\%), glutamic acid decarboxylase anti- 
body (GADA) in 21 patients (46.7\%), and insulin autoantibody in 14 patients $(31.1 \%)$. Six patients $(13.3 \%)$ were positive for both ICA and GADA. One patient (2.2\%) tested positive for all three antibodies.

When the data were compared during a period of 3 to 6 months before and after the COVID-19 outbreak, no significant difference was found in average BMI $\left(21.62 \pm 6.17 \mathrm{~kg} / \mathrm{m}^{2}\right.$ vs. $21.06 \pm 4.97$ $\left.\mathrm{kg} / \mathrm{m}^{2}, p=0.250\right)$. The HbAlc levels were significantly increased after the COVID-19 outbreak (before, $7.70 \% \pm 1.38 \%$ vs. after, $8.30 \% \pm 2.05 \% ; p=0.012$ ) (Table 1 ). HbAlc levels increased significantly after COVID-19 and tended to be higher in the pubertal group (before, $7.83 \% \pm 1.56 \%$ vs. after, $8.46 \% \pm 2.40 \%$; $p=0.025$ ) than in the prepubertal group (before, $7.03 \% \pm 0.90 \%$ vs. after, $7.23 \% \pm 1.24 \%$; $p=0.565$ ).

\section{Comparison of diabetic ketoacidosis severity in patients with newly diagnosed type 1 diabetes mellitus}

There were 10 age-matched patients newly diagnosed with T1DM before the onset of the COVID-19 outbreak and 10 patients diagnosed after the outbreak. The frequency of DKA tended to be higher during puberty (Fig. 1). The COVID-19 outbreak period was spring and early summer, and the patients in the age-matched control group before COVID-19 visited the hospital at 2:4:2:2 in spring, summer, autumn, and winter.

The 10 newly diagnosed patients before the outbreak comprised one male and nine female subjects, with a median age of 12.0 years. Four patients were confirmed with DKA at the time of admission, of whom two patients showed severe clinical symptoms such as altered mental status, dyspnea, and tachypnea. Based on DKA severity, patients were classified as follows: two patients had mild disease, and two patients had moderate disease. Two patients required care from the pediatric intensive care unit (PICU).
After the outbreak, there were 10 children with newly diagnosed T1DM (six males and four females), with a median age of 11.5 years. Six patients had DKA at the time of admission, of whom four showed severe symptoms. When classified according to

Table 1. Baseline characteristics and comparison of BMI and HbA1c in patients with T1DM 3-6 months before and after COVID-19 pandemic

\begin{tabular}{lc}
\hline Characteristic & Data \\
\hline No. of patients & 45 \\
Age (yr) & $15.83 \pm 6.13$ \\
Sex, male:female & $23: 22$ \\
Initial random glucose (mg/dL) & $413.53 \pm 202.62$ \\
C-peptide (ng/mL) & $0.65 \pm 0.56$ \\
Patients with identified autoantibodies & \\
ICA & $2(4.4)$ \\
GADA & $21(46.7)$ \\
IAA & $14(31.1)$ \\
ICA+GADA & $6(13.3)$ \\
ICA+GADA + IAA & $1(2.2)$ \\
Autoantibody negative & $8(17.8)$ \\
BMI (kg/m²) & \\
Before COVID-19 & $21.62 \pm 6.17$ \\
After COVID-19 & $21.06 \pm 4.97$ \\
p-value & 0.250 \\
HbA1c (\%) & \\
Before COVID-19 & $7.70 \pm 1.38$ \\
After COVID-19 & $8.30 \pm 2.05$ \\
p-value & $0.012^{\text {a) }}$ \\
\hline
\end{tabular}

Values are presented as number only, mean \pm standard deviation, or number (\%).

$\mathrm{BMI}$, body mass index; $\mathrm{HbA1c}$, hemoglobin A1c; T1DM, type 1 diabetes mellitus; COVID-19, coronavirus disease 2019; ICA, islet cell antibody; GADA, glutamic acid decarboxylase antibody; IAA, insulin autoantibody. a) $p<0.05$.

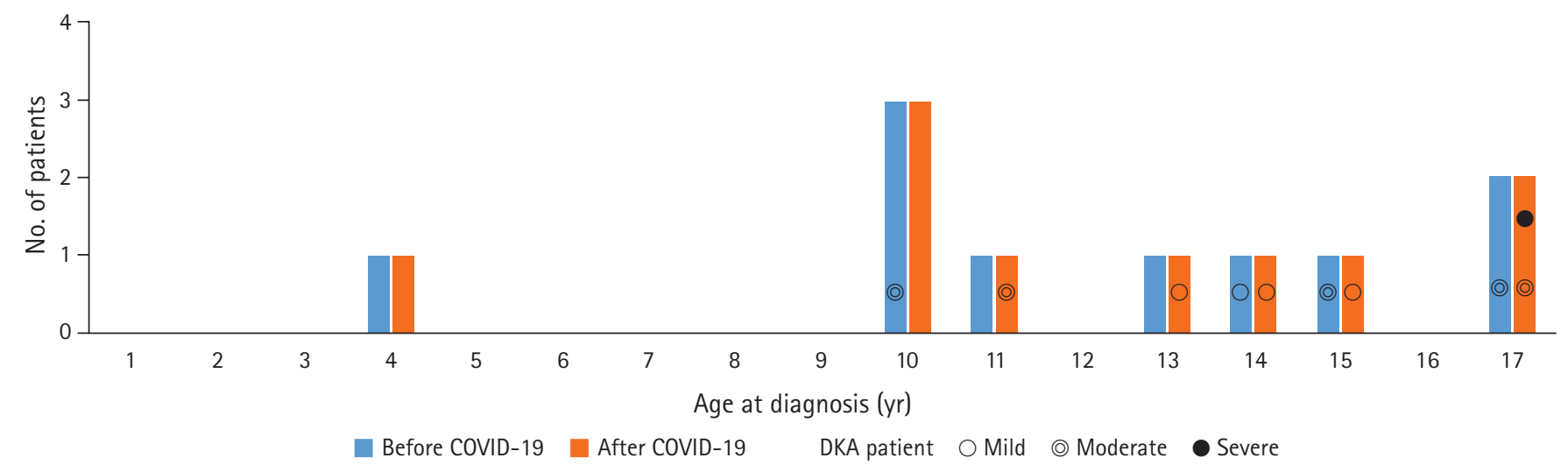

Fig. 1. Distribution by age of newly diagnosed type 1 diabetes mellitus patients before and after the coronavirus disease 2019 (COVID-19) outbreak. Non-diabetic ketoacidosis (DKA) patients were not indicated, and DKA patients were indicated according to severity. 
DKA severity, three patients had mild disease, two had moderate disease, one patient had severe disease, and three patients required PICU care (Fig. 2).

$\mathrm{HbA1c}$ levels of newly diagnosed T1DM patients were significantly higher in patients hospitalized after the COVID-19 outbreak than in those hospitalized before the outbreak (before, $11.15 \%$ vs. after, $13.60 \% ; p=0.036$ ) (Table 2). Moreover, random serum glucose levels were higher in patients with T1DM after COVID-19 (before, $300 \mathrm{mg} / \mathrm{dL}$ vs. after, $371 \mathrm{mg} / \mathrm{dL} ; p=0.203$ ). The levels of $\mathrm{pH}$ and bicarbonate were lower after COVID-19, but not statistically significant ( $\mathrm{pH}$ : before, 7.40 vs. after, 7.25 , $p=0.333$; bicarbonate: before, $19.50 \mathrm{mmol} / \mathrm{L}$ vs. after, 10.50 $\mathrm{mmol} / \mathrm{L}, p=0.221$ ).

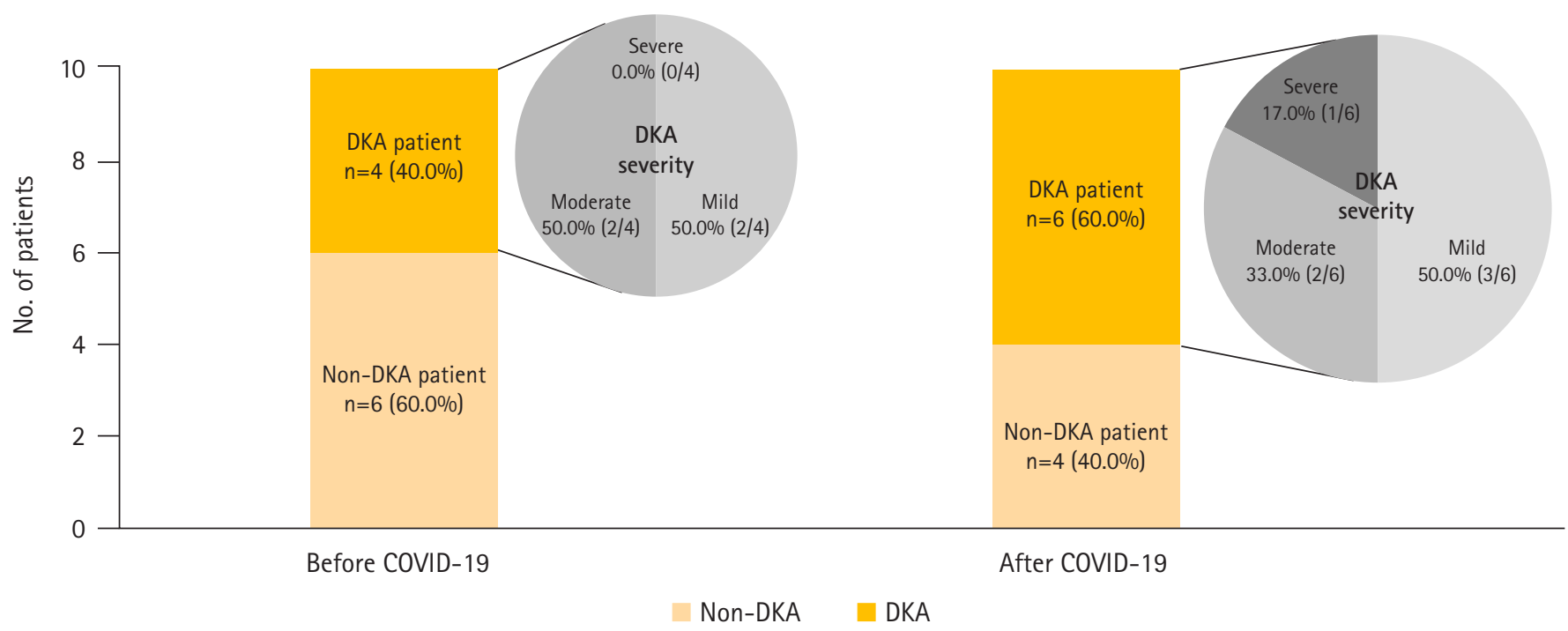

Fig. 2. Comparison of ratio of diabetic ketoacidosis (DKA) and DKA severity in newly diagnosed type 1 diabetes mellitus patients hospitalized before and after the coronavirus disease 2019 (COVID-19) outbreak.

Table 2. Comparison of HbA1c in newly diagnosed T1DM patients hospitalized before and after the COVID-19 outbreak

\begin{tabular}{lccc}
\hline Variable & Before COVID-19 & After COVID-19 & $p$-value \\
\hline No. of patients & 10 & 10 & 0.317 \\
DKA (mild:moderate:severe) & $4(2: 2: 0)$ & $6(3: 2: 1)$ & 0.439 \\
Age (yr) & $12.0(4.0-17.0)$ & $11.5(3.90-17.0)$ & 0.414 \\
Sex, male:female & $1: 9$ & $6: 4$ & $0.036^{\text {a) }}$ \\
HbA1c $(\%)$ & $11.15(7.40-14.70)$ & $13.60(11.70-16.00)$ & 0.203 \\
Glucose (mg/dL) & $300(176-608)$ & $371(116-915)$ & 0.333 \\
pH & $7.40(7.06-7.44)$ & $7.25(6.83-7.45)$ & 0.221 \\
Bicarbonate (mmol/L) & $19.50(5-26)$ & $10.50(2-25)$ &
\end{tabular}

Values are presented as number only or median (range).

HbA1c, hemoglobin A1c; T1DM, type 1 diabetes mellitus; COVID-19, coronavirus disease 2019; DKA, diabetic ketoacidosis. a) $p<0.05$. 
connection with the Great East Japan Earthquake Disaster, HbAlc levels did not increase significantly by actively controlling the drugs through regular outpatient visits [18]. However, the effects of the COVID-19 pandemic on glycemic control in patients with $\mathrm{DM}$ and patient management guidelines are not well established. In India, a total lockdown was implemented for 21 days, from March 24, 2020, to prevent the spread of COVID-19; a systematic study revealed that blood sugar levels and DM-related complications were increased during this period [19]. The present study also demonstrated a significant increase in $\mathrm{HbAlc}$ levels in children with T1DM after the outbreak. Therefore, we suggest a possible explanation for this phenomenon. Wang et al. [20] reported a decrease in physical activity during the COVID-19 outbreak in students due to limitations in daily life. It has already been reported that students are less physically active, have more screen time, and display irregular sleeping patterns when they are not attending school, such as during weekends and breaks [21,22]. As such, there were restrictions on daily life, including school attendance, as observed in this study, which led to a decrease in physical activity and changes in lifestyle habits. In fact, patients and their guardians at the outpatient clinic cited irregular lifestyle habits as the cause of the increase in blood sugar levels. Moreover, there were no significant changes in BMI, suggesting that the increase in blood sugar level was due to irregular lifestyle changes rather than increased food intake. MacMillan et al. [23] reported that these lifestyle changes have a detrimental impact on blood sugar control. In addition, Nansel et al. [24] reported that dietary control, such as intake of foods with high fiber and low glycemic index and carbohydrate-containing foods improves glycemic control, suggesting that irregular eating habits due to lifestyle changes cause an increase in blood sugar levels. Furthermore, irregular eating habits may interfere with regular insulin injections. Verma et al. [25] reported that COVID-19 lockdown resulted in an increase in blood sugar levels in patients with T1DM because of the difficulty in prescribing blood sugar measuring devices and insulin injections. In this study, there were no cases in which children could not receive insulin treatment due to the unavailability of insulin shots. However, this may be because the insulin dose was not properly controlled by checking blood sugar levels due to irregular lifestyles.

In this study regarding the frequency and severity of DKA in newly diagnosed patients, the $\mathrm{HbAlclevels}$ and DKA severity increased after COVID-19. The frequency and severity of DKA increased compared to the previous age-matched patients. In a study conducted by Rabbone et al. [26] in 53 clinics in Italy, it was observed that the proportion of newly diagnosed T1DM patients with severe DKA significantly increased from $44.3 \%$ in 2020 to
$36.1 \%$ in $2019(p=0.030)$. A German study conducted by Kamrath et al. [27] also showed that the rates of DKA and severe DKA were higher during the COVID-19 outbreak. In the case of patients with newly diagnosed DM in this study, even when symptoms such as polyurea and polyphagia occurred, because of concerns about infection when visiting a medical institution or when going outside, the patients did not visit the hospital until the disease progressed and the symptoms were severe enough to cause problems in their daily life, such as dyspnea and altered mental status. Moreover, we analyzed the cause of the increased DKA severity before and after the COVID-19 outbreak. In Korea, schools conduct health checkups for all students in the spring, among which, urine analysis is conducted for the 1st and 4th grades of elementary school, the 1st grades of middle school, and the 1st grades of high school [28]. Due to the COVID-19 outbreak in the Daegu-Gyeongbuk area, there were daily life restrictions and delays in school attendance and admissions. During that time, students were unable to attend schools for a considerable period of time to prevent infection during the COVID-19 pandemic; hence, they were unable to undergo health checkups, as this period fell from March to April, which marks the beginning of a new school year in Korea. Furthermore, 1.4\% of the students had high blood sugar levels according to the 2019 Student Health Examination Sample Statistics [28]. As such, most of the patients with newly diagnosed DM who have no symptoms or show mild symptoms are identified during school checkups and are asked to visit the hospital. In this study, health checkups in school were not possible during the COVID-19 outbreak. In the control group before the COVID-19 outbreak, two out of 10 patients were referred for hospital treatment due to abnormal findings in the screening test and were diagnosed with T1DM when symptoms were mild. School screening is useful for detecting type $2 \mathrm{DM}$ with relatively mild symptoms and a long latent period of asymptomatic progression. However, it is meaningful because it can be detected through school examinations in the early stage of type $1 \mathrm{DM}$, when symptoms are not severe. This COVID-19-induced lockdown led to the omission of screening, and the symptoms were recognized only after the onset of severe DKA.

If symptoms of DM occur in both newly diagnosed patients and previously diagnosed patients, it is important to visit a hospital and check the progress before the symptoms worsen. Regular glucose monitoring and insulin medication are important for glycemic control in pediatric T1DM patients after the acute symptoms have improved. For this, it is important to check the progress of periodic outpatient visits [29]. In an Italian study conducted by Tornese et al. [30], there were cases of good glycemic control in patients with T1DM through continuous glucose monitoring (CGM) and insu- 
lin pump along with physical activity and diet control through telemedicine. This study is limited by the lack of statistical data on the reasons for poor blood glucose control. Specifically, there were no studies on the activity, total intake, and insulin dose of the patients. The daily insulin dose, number of daily blood sugar test checks, and rate of CGM application were not investigated. Further research is needed, and more careful patient observation is required while following general DM guidelines during the COVID-19 epidemic. This includes checking the readings of blood sugar and insulin doses through frequent and regular follow-up and monitoring of physical activity and diet.

This retrospective chart review was conducted at a single center. When searching for an age-matched control group for newly diagnosed T1DM patients in the COVID-19 period, patients who visited the hospital in the closest season as possible were selected, but it was difficult to completely match and analyze patients by season by performing a single-center study with small sample size. Further multicenter analysis with a larger number of patients will be needed to identify factors in addition to COVID-19 that may have an influence, such as seasonality. Gender was also considered, but it was difficult to match because of the small number of patients. One more limitation is the lack of data on patients who did not visit the hospital within the study period. However, as the data were collected from a region with a severe COVID-19 outbreak, we believe that this study will be helpful in examining the incidence rate, symptom severity, and changes in the prevalence rate and degree of glycemic control of DM in children and adolescents.

In conclusion, this study reports the incidence of increased blood sugar levels and newly diagnosed cases of T1DM in children with severe DKA due to the COVID-19 situation in the Daegu-Gyeongbuk area. In the future, a large-scale, long-term follow-up study and active blood sugar management in patients with T1DM are required during the COVID-19 pandemic.

\section{Notes}

\section{Conflicts of interest}

No potential conflict of interest relevant to this article was reported.

\section{Author contributions}

Conceptualization: MSL, CWK, JEM; Data curation: MSL, RL, JEM; Formal analysis: MSL; Investigation, Software, Validation: MSL, JEM; Writing-original draft: MSL, RL; Writing-review \& editing: CWK, JEM.

\section{ORCID}

Mi Seon Lee, https://orcid.org/0000-0001-9441-8018

Rosie Lee, https://orcid.org/0000-0003-3285-3916

Cheol Woo Ko, https://orcid.org/0000-0002-0643-7233

Jung Eun Moon, https://orcid.org/0000-0001-9786-7898

\section{References}

1. Lu H, Stratton CW, Tang YW. Outbreak of pneumonia of unknown etiology in Wuhan, China: the mystery and the miracle. J Med Virol 2020;92:401-2.

2. Hui DS, I Azhar E, Madani TA, Ntoumi F, Kock R, Dar O, et al. The continuing 2019-nCoV epidemic threat of novel coronaviruses to global health: the latest 2019 novel coronavirus outbreak in Wuhan, China. Int J Infect Dis 2020;91:264-6.

3. Huang C, Wang Y, Li X, Ren L, Zhao J, Hu Y, et al. Clinical features of patients infected with 2019 novel coronavirus in $\mathrm{Wu}$ han, China. Lancet 2020;395:497-506.

4. World Health Organization (WHO). Timeline: WHO's COVID-19 response [Internet]. Geneva: WHO; 2020 [cited 2020 Sep 21]. https://www.who.int/emergencies/diseases/ novel-coronavirus-2019/interactive-timeline\#!

5. Kim JY, Choe PG, Oh Y, Oh KJ, Kim J, Park SJ, et al. The first case of 2019 novel coronavirus pneumonia imported into Korea from Wuhan, China: implication for infection prevention and control measures. J Korean Med Sci 2020;35:e61.

6. Kim JH. An JA, Min P, Bitton A, Gawande AA. How South Korea responded to the Covid-19 outbreak in Daegu. NEJM Catal Innov Care Deliv 2020;1(4):10.1056/CAT.20.0159.

7. Lee JY, Hong SW, Hyun M, Park JS, Lee JH, Suh YS, et al. Epidemiological and clinical characteristics of coronavirus disease 2019 in Daegu, South Korea. Int J Infect Dis 2020;98:462-6.

8. Yang X, Yu Y, Xu J, Shu H, Xia J, Liu H, et al. Clinical course and outcomes of critically ill patients with SARS-CoV-2 pneumonia in Wuhan, China: a single-centered, retrospective, observational study. Lancet Respir Med 2020;8:475-81.

9. Zhou J, Tan J. Diabetes patients with COVID-19 need better blood glucose management in Wuhan, China. Metabolism 2020;107:154216.

10. Kim MK, Jeon JH, Kim SW, Moon JS, Cho NH, Han E, et al. The clinical characteristics and outcomes of patients with moderate-to-severe coronavirus disease 2019 infection and diabetes in Daegu, South Korea. Diabetes Metab J 2020;44:602-13.

11. Cardona-Hernandez R, Cherubini V, Iafusco D, Schiaffini R, Luo X, Maahs DM. Children and youth with diabetes are not at increased risk for hospitalization due to COVID-19. Pediatr Diabetes 2021;22:202-6. 
12. International Society for Pediatric and Adolescent Diabetes (ISPAD). Coronavirus infection (COVID-19)-II ISPAD summary [Internet]. Berlin: ISPAD; 2020 [cited 2020 Sep 26]. https:// www.ispad.org/page/CoronavirusinfectionCOVID-19-IIISPADSummary.

13. Wolfsdorf JI, Glaser N, Agus M, Fritsch M, Hanas R, Rewers A, et al. ISPAD Clinical Practice Consensus Guidelines 2018: diabetic ketoacidosis and the hyperglycemic hyperosmolar state. Pediatr Diabetes 2018;19(Suppl 27):155-77.

14. Fonseca VA, Smith H, Kuhadiya N, Leger SM, Yau CL, Reynolds K, et al. Impact of a natural disaster on diabetes: exacerbation of disparities and long-term consequences. Diabetes Care 2009;32:1632-8.

15. Inui A, Kitaoka H, Majima M, Takamiya S, Uemoto M, Yonenaga C, et al. Effect of the Kobe earthquake on stress and glycemic control in patients with diabetes mellitus. Arch Intern Med 1998; 158:274-8.

16. Kirizuka K, Nishizaki H, Kohriyama K, Nukata O, Arioka Y, Motobuchi M, et al. Influences of The Great Hanshin-Awaji Earthquake on glycemic control in diabetic patients. Diabetes Res Clin Pract 1997;36:193-6.

17. Sengül A, Ozer E, Salman S, Salman F, Sağlam Z, Sargin M, et al. Lessons learnt from influences of the Marmara earthquake on glycemic control and quality of life in people with type 1 diabetes. Endocr J 2004;51:407-14.

18. Nishikawa Y, Fukuda Y, Tsubokura M, Kato S, Nomura S, Saito Y. Managing type 2 diabetes mellitus through periodical hospital visits in the aftermath of the Great East Japan Earthquake disaster: a retrospective case series. PLoS One 2015;10:e012 5632.

19. Ghosal S, Sinha B, Majumder M, Misra A. Estimation of effects of nationwide lockdown for containing coronavirus infection on worsening of glycosylated haemoglobin and increase in diabetes-related complications: a simulation model using multivariate regression analysis. Diabetes Metab Syndr 2020;14:319_ 23.

20. Wang G, Zhang Y, Zhao J, Zhang J, Jiang F. Mitigate the effects of home confinement on children during the COVID-19 outbreak. Lancet 2020;395:945-7.

21. Brazendale K, Beets MW, Weaver RG, Pate RR, Turner-McGrievy GM, Kaczynski AT, et al. Understanding differences be- tween summer vs. school obesogenic behaviors of children: the structured days hypothesis. Int J Behav Nutr Phys Act 2017; 14: 100.

22. Wang G, Zhang J, Lam SP, Li SX, Jiang Y, Sun W, et al. Ten-year secular trends in sleep/wake patterns in Shanghai and Hong Kong school-aged children: a tale of two cities. J Clin Sleep Med 2019;15:1495-502.

23. MacMillan F, Kirk A, Mutrie N, Matthews L, Robertson K, Saunders DH. A systematic review of physical activity and sedentary behavior intervention studies in youth with type 1 diabetes: study characteristics, intervention design, and efficacy. Pediatr Diabetes 2014;15:175-89.

24. Nansel TR, Lipsky LM, Liu A. Greater diet quality is associated with more optimal glycemic control in a longitudinal study of youth with type 1 diabetes. Am J Clin Nutr 2016;104:81-7.

25. Verma A, Rajput R, Verma S, Balania VK, Jangra B. Impact of lockdown in COVID 19 on glycemic control in patients with type 1 diabetes mellitus. Diabetes Metab Syndr 2020;14:12136.

26. Rabbone I, Schiaffini R, Cherubini V, Maffeis C, Scaramuzza A; Diabetes Study Group of the Italian Society for Pediatric Endocrinology and Diabetes. Has COVID-19 delayed the diagnosis and worsened the presentation of type 1 diabetes in children? Diabetes Care 2020;43:2870-2.

27. Kamrath C, Mönkemöller K, Biester T, Rohrer TR, Warncke K, Hammersen J, et al. Ketoacidosis in children and adolescents with newly diagnosed type 1 diabetes during the COVID-19 pandemic in Germany. JAMA 2020;324:801-4.

28. Korean Ministry of Education. 2019 Student health test sample statistics results [Internet]. Sejong, KR: Korean Ministry of Education; 2019 [cited 2020 Sep 22]. https://www.moe.go.kr/ boardCnts/view.do?boardID $=294 \&$ lev $=0$ \&statusYN $=$ $\mathrm{W} \& \mathrm{~s}=$ moe\&m $=0204 \&$ opType $=\mathrm{N} \&$ boardSeq $=81310$.

29. Hood KK, Peterson CM, Rohan JM, Drotar D. Association between adherence and glycemic control in pediatric type 1 diabetes: a meta-analysis. Pediatrics 2009; 124:e1171-9.

30. Tornese G, Ceconi V, Monasta L, Carletti C, Faleschini E, Barbi E. Glycemic control in type 1 diabetes mellitus during COVID-19 quarantine and the role of in-home physical activity. Diabetes Technol Ther 2020;22:462-7. 